\title{
From the cell to the community: AIDS research in California
}

\author{
The University of California Universitywide AIDS Research Program annual meeting, February 2000
}

\section{INTRODUCTION}

Between 1981 and 1999, more than 110,00 cases of the acquired immunodeficiency syndrome (AIDS) have been reported in California, of whom nearly 70,000 patients have died. Since 1983, the University of California has funded basic, clinical, social, and behavioral research on the human immunodeficiency virus (HIV)/AIDS through the Universitywide AIDS Research Program (UARP). Major accomplishments of UARP-supported research include the isolation and characterization of HIV, the detection and elimination of HIV in blood clotting factor VIII, and in early studies, the demonstration of the rapid production and turnover of HIV in the blood of infected persons.

Together with its advisory group, the Universitywide Task Force on AIDS, UARP convenes an annual meeting that provides an opportunity for the exchange of new information on HIV/AIDS in California. Participants include bench scientists, clinicians, policymakers, outreach workers, community-based organizations, and members of affected communities. Most of the studies presented at the UARP conference are in their early stages and modestly funded. While some go on to receive larger federal grants, others are quickly integrated into new interventions or investigations.

In this issue of wjm, we share highlights of the 3rd Annual Conference on AIDS Research in California. $\dagger$ The plenary theme for the year 2000 was "Early Events and Intervention in HIV Infection." Although the recent and ongoing advances in highly active antiretroviral therapy are well known, the meeting provided a fertile venue for presenting other innovative areas for treatment and prevention.

\section{FEATURED SPEAKER HIGHLIGHTS Primary HIV infection in high-risk populations: the options project}

\section{Presenter: James 0 Kahn}

Primary HIV infection refers to the clinical events that occur within 12 months of becoming infected. Clinicians should think of primary HIV infection as a diagnosis in patients who may have been exposed to the virus and who

*Affiliations are listed on p 124

$\dagger$ More detailed descriptions of each conference project, including quantitative data, are contained in the Compendium of Abstracts (available at www.ucop.edu/srphome/uarp) or on request to Universitywide AIDS Research Program, 300 Lakeside Dr, 6th floor, Oakland, CA 94612 3550, attn: Denise Elkins.

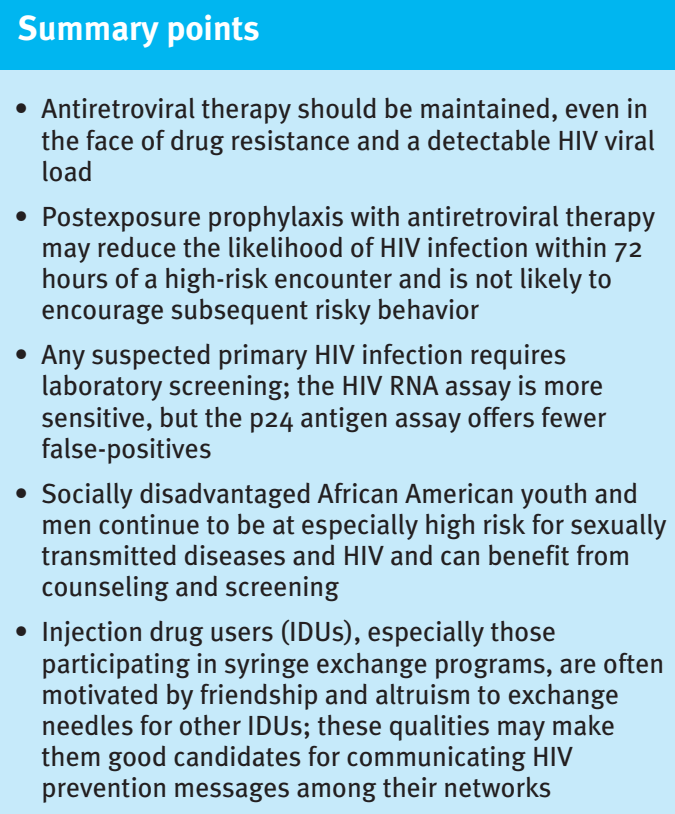

- Antiretroviral therapy should be maintained, even in the face of drug resistance and a detectable HIV viral load

- Postexposure prophylaxis with antiretroviral therapy may reduce the likelihood of HIV infection within 72 hours of a high-risk encounter and is not likely to encourage subsequent risky behavior

- Any suspected primary HIV infection requires laboratory screening; the HIV RNA assay is more sensitive, but the p24 antigen assay offers fewer false-positives

- Socially disadvantaged African American youth and men continue to be at especially high risk for sexually transmitted diseases and HIV and can benefit from counseling and screening

- Injection drug users (IDUs), especially those participating in syringe exchange programs, are often motivated by friendship and altruism to exchange needles for other IDUs; these qualities may make them good candidates for communicating HIV prevention messages among their networks

present with rash, fever, myalgia, pharyngitis, or other features of a viral illness. Such patients should be screened for HIV-1 RNA and antibody using the enzyme-linked immunosorbent assay (EIA). Early therapy with protease inhibitors used in combination with other antiretroviral agents can control HIV during the earliest, acute phase of infection. However, identifying individuals in the community who have recently become infected is very difficult.

Patients with primary HIV infection are at high risk of transmitting HIV infection to others. In the absence of antiretroviral therapy, levels of HIV RNA are often very high during acute HIV infection and can reach above $10^{6}$ copies per milliliter. Previous studies suggest that the level of HIV RNA is important in transmission.

The Options Project, a collaboration of University of California scientists, exemplifies UARP's approach of targeting research "from the cell to the community." This research brings together basic, clinical, and behavioral scientists who are interested in primary HIV infection. The collaboration addresses a number of key questions (see box).

In the Options Project cohort study, patients with signs or symptoms of primary HIV infection are screened to assess their HIV antibody and viral RNA status. Patients either refer themselves, or they are referred by clinicians, community-based organizations, emergency departments, or HIV test centers. Enrollment into the study is
Nancy E Moss*

George Lemp

Ricky Bluthenthal

Mark A Goldsmith Richard Haubrich James 0 Kahn

Correspondence to:

Dr Lemp

George.Lemp@ucop.edu

West J Med

2000;173:119-124 


\section{The Options Project: key research questions}

- What are the key ways that people present with HIV?

- In what ways are infected people different from the uninfected?

- How can we prevent HIV infection?

- Among newly infected persons, what are the recent virologic events and can they predict later events?

- What are the immune responses during infection?

- How can we intervene toward better outcomes?

ongoing. The project at the San Francisco General Hospital identifies newly infected patients and brings them into primary care while also observing patients who erroneously believe that they are infected. Most participants are men who have sex with men, and more than half have a history of substantial alcohol and drug use.

Of 310 patients screened to date, 122 with primary HIV infection have been identified. Infected participants were more likely to have had high-risk sexual behaviors than those who were uninfected. They were also more likely to have had depression and to have had a history of sexually transmitted diseases (STDs). One third of patients with primary HIV infection believed that combination therapy for HIV would prevent transmission to an uninfected person.

The Options Project has also monitored whether the viral strains in primary HIV infection are remaining susceptible to antiretroviral therapy. Although the number of isolates was relatively small, the proportion of patients whose HIV strain was resistant to zidovudine fell from $16 \%$ in 1996 to $3 \%$ in 1999 , whereas the proportion resistant to protease inhibitors increased from $0 \%$ to $7.7 \%$ during the same period. Monitoring those with acute HIV infection remains an optimal method of assessing trends in the transmission of resistant virus.

The project team has evaluated patients with primary HIV infection or high-risk sexual behaviors during the course of their acute infection. With the benefit of counseling interventions in this cohort study, the practice of insertive anal intercourse with or without a condom decreased, but $50 \%$ of respondents continued to report high-risk activities. Providing early antiretroviral therapy to prevent immune destruction in patients identified with primary HIV infection is necessary but not sufficient to eliminate the virus. Behavioral modification strategies must also be included to reduce the risk of HIV transmission.

Further reading

Hecht FM, Rawal BD, Kahn JO, et al. Diagnosis of acute HIV infection using p24 antigen and HIV-1 RNA assays. 6th Conference on
Retroviruses and Opportunistic Infections 1999, Jan 31-Feb 4;101. Abstract 178.

Rawal BD, Hecht FM, Kahn JO, et al. Dual EIA test strategy for enrolling persons with recent HIV infection into a primary HIV treatment trial. 6th Conference on Retroviruses and Opportunistic Infections 1999, Jan 31-Feb 4;159. Abstract 486.

\section{Treating high-risk populations with postexposure prophylaxis \\ Presenter: Mitchell H Katz}

Postexposure prophylaxis may be one way of reducing new infections among persons who have engaged in a risk behavior with someone known to be or suspected of being infected with HIV. Postexposure prophylaxis involves providing combination antiretroviral therapy (Combivir [zidovudine, $300 \mathrm{mg}$, and lamivudine, $150 \mathrm{mg}$ ) to exposed persons within 72 hours of the initial exposure for a 28day course.

A recently completed project in San Francisco is addressing the feasibility of postexposure prophylaxis. This nonrandomized observational study enrolled 401 persons who sought postexposure prophylaxis, more than $90 \%$ of them for a sex-related exposure. Most participants were aged 31 to 50 years, $90 \%$ were men, and $69 \%$ were white. The cohort was observed and assessed at 1, 2, 4, and 26 weeks after intake. Risk reduction and medication adherence counseling was provided at several sessions, and patients were encouraged to bring in source partners for assessment.

Postexposure prophylaxis is useful only if the source partner is HIV-positive, but the partner's serostatus was unknown to $57 \%$ of participants. Clinicians should use community levels of HIV prevalence in deciding whether postexposure prophylaxis is appropriate for patients. The most important issue in this decision is the likely risk factors of the source partner, if known.* In areas such as San Francisco and Los Angeles, postexposure prophylaxis is advisable if the source is an IDU or a man who has sex with men because the prevalence of HIV in these cities is $5 \%$ to $10 \%$ in IDUs and $20 \%$ to $30 \%$ in men who have sex with men.

Patients using postexposure prophylaxis experience considerable subjective toxic reactions-nausea, anorexia, headache, fatigue - but virtually no safety issues, as demonstrated by laboratory values. From a public health perspective, postexposure prophylaxis is less cost-effective than behavioral counseling or condom distribution, but from a clinical perspective, it makes sense and it does not appear to enhance behavioral risk. Postexposure prophylaxis provides an opportunity to work with patients on current and future risk status, to evaluate for other STDs,

\footnotetext{
*The absolute prevalence level at which postexposure prophylaxis should be prescribed is unclear, although many physicians suggest between $1 \%$ and $5 \%$.
} 
and to immunize for hepatitis B. These patients do not want to be infected and are highly motivated to examine their behavior.

Serious questions still facing the project team include: how can clinicians encourage use of postexposure prophylaxis among racial and ethnic minorities (only 18\% of patients seeking postexposure prophylaxis have been either Latino or African American) or among IDUs? Is it efficacious at preventing HIV infection? How can we prove it? Such challenges are to be expected of any HIV prevention strategy.

Further reading

Katz MH, Gerberding JL. The care of persons with recent sexual exposure to HIV. Ann Intern Med 1998;128:306-312.

Katz MH, Gerberding JL. Postexposure treatment of persons exposed to HIV through sexual contact or injection drug use. $N$ Engl J Med 1997;336:1097-1100

\section{Viral fusion: the scientific underpinnings of early HIV infection}

\section{Presenter: Eric Hunter}

Although several kinds of antiretroviral therapies are currently available, cellular entry by HIV is a compelling target for a new class of antiretroviral agents.

Three possible targeting strategies may lead to new therapeutics directed against viral entry into the cell:

- Disrupting binding of CD4 (a molecule on the helper T-cell surface) to gp120 (the membrane protein of HIV) through direct antagonism at the interface because the CD4 protein serves as a gatekeeper to viral entry into the cell.

- Preventing engagement of a cellular chemokine receptor or "coreceptor" through agents that bind to key sites on the coreceptor, the primary cellular cofactor for HIV entry.

- Interfering with fusion by using peptides or small molecules to disrupt the structure or dynamics of gp41 (the exterior or envelope protein of the virus that facilitates fusion with the cell).

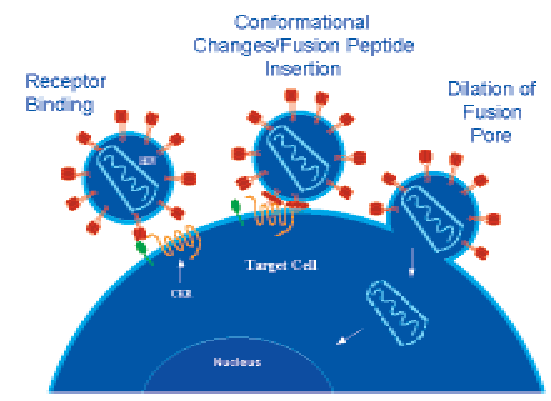

Conformational changes in envelope allow cellular entry by HIV
The third strategy may be the most promising for blocking viral entry into the cell and is now being tested in clinical studies.

In a Phase I/IIB clinical trial, researchers at the outpatient clinic of the University of Alabama, Birmingham, Medical Center gave a prototypical peptide antagonist of HIV entry intravenously twice a day in the clinic to 16 HIV-infected patients in 4 dose groups (3, 10, 30, and $100 \mathrm{mg}$ twice a day) for 14 days. Patients had a dosedependent reduction in viral load, achieving as high as a 2-log effect, suggesting that HIV could be reduced to preclinical levels. Frequent intravenous administration may not be necessary; intermittent subcutaneous infusions may effectively reduce viral load. These studies suggest that viral fusion is a viable target for the design and development of new treatment strategies. They also illustrate the way in which basic research may have immediate clinical implications.

Second-generation peptides with improved pharmacologic properties are also undergoing clinical trials. Meanwhile, a major goal is the development of small-molecule antagonists that will similarly disrupt the viral fusion process. Initial clinical tests of fusion inhibitors suggest that disrupting the binding process will be clinically useful.

Further reading

Kilby JM, Hopkins S, Venetta TM, et al. Potent suppression of HIV-1 replication in humans by T-20, a peptide inhibitor of gp 41 mediated virus entry. Nat Med 1998;4:1302-1306.

Moore JP, Trkola A, Dragic T. Co-receptors for HIV-1 entry. Curr Opin Immunol 1997;9:551-562.

Richman DD. Nailing down another HIV target. Nat Med 1998;4:1232-1233.

\section{CURRENT QUESTIONS IN HIV/AIDS RESEARCH IN CALIFORNIA \\ Clinical sciences}

Progress in clinical research has resulted in an increase in survival and quality of life for people with HIV/AIDS. A current priority is to unite the growing understanding of the molecular underpinnings of the virus and the increased use of antiretroviral drugs with progress in diagnosis and treatment. Changes in treatment have led to 5 important clinical questions.

1. Which laboratory tests and symptoms are most predictive of primary HIV infection?

Symptomatic $(n=436)$ and asymptomatic $(n=69)$ subjects with recent exposure to possibly HIV-infected persons were screened for primary HIV infection, and their symptoms were recorded. Researchers compared 2 different methods of detecting recent HIV seroconversion. Plasma HIV RNA testing was compared with p24 antigen (p24 is a structural protein of HIV) in differentiating primary HIV infection in those whose 
ELISA screens were negative or whose Western blot screens were indeterminate (these 2 are standard HIV screens). The p24 antigen was almost as sensitive as the HIV RNA test and had the extra advantage of fewer falsepositives in patients ultimately found to be negative for HIV.

The following symptoms occur more commonly in patients with primary HIV infection than in those who are uninfected or chronically infected: fever, myalgia, arthralgia, rash, night sweats, and pharyngitis $(P<0.05)$. But no constellation of symptoms was sufficiently sensitive or specific to predict primary HIV infection without laboratory screening.

2. Why do a subset of patients with primary HIV infection have reduced antiviral drug susceptibility?

One theory is that these patients share a common source of infection, perhaps because they come from the same region. But preliminary results on 49 patients, for whom therapy with 1 class of antiretroviral agents failed, provide no evidence for this theory. Using molecular epidemiologic techniques, the authors found no evidence suggesting clustering of viral nucleotide sequences, and they concluded that the cases did not appear to be linked by person, place, or time.

3. What are the pros and cons of interrupting antiretroviral combination therapy?

Should antiretroviral therapy be continued even if there is evidence of viral failure? A randomized study of 18 patients showed that the continuation of antiretroviral therapy has clear benefit, even if it appears not to be working well, as evidenced by increased viral load. Patients who were continued on therapy were often found to maintain stable CD4 cell counts despite virologic failure. For those discontinuing antiretroviral therapy, CD4 counts fell substantially by 12 weeks, in addition to an increase in viral load. These results suggest that continuing protease inhibitor-based antiretroviral therapy, despite the apparent treatment failure indicated by a rebound in viral load, has a beneficial effect in many patients because the therapy selects for less virulent HIV strains. The investigators hypothesized that this is possibly due to a weakened virus; thus, when the virus mutates and becomes resistant to protease inhibitor-based therapy, it does so at the cost of reduced virulence.

4. Can a Web-based surveillance system be useful for ascertaining changes in response to treatment?

A team at San Francisco General Hospital is observing a prospective, observational cohort of patients receiving care from the Positive Health Program (PHP) using a database on the Web. The goal is to provide rapid, "realworld" evaluation of current treatment guidelines. The high-need PHP population is $17 \%$ women, $5 \%$ of the patients are unstably housed, and 65\% receive Medi-Cal or Medicare. The patient group is $50 \%$ white, $30 \%$ black,
15\% Latino, and 5\% Asian. Between 1996 and 1998, the mean CD4 cell count increased. However, more than half of the patients had never achieved consecutive HIV RNA levels below the limits of detection. This could be an indication that for a large proportion of patients in clinical practice, current antiretroviral regimens may be failing and that the prevalence of HIV resistance to agents might be high.

5. How do characteristics of the HIV-1 envelope gene affect perinatal transmission?

A better understanding of how HIV-1 is transmitted to infants will guide the development of preventive therapy. A preliminary study suggests that there are differences in the level or degree of diversity of the HIV-1 envelope gene between infected mothers who do $(\mathrm{n}=23)$ and those who do not $(n=36)$ transmit HIV to their infants in utero versus intrapartum. Researchers measured HIV-1 envelope gene diversity in women during gestation and at delivery and, in infants, at the first time testing positive and at age 12 weeks. They were able to sequence the gene and group its variants by frequency, showing that gene diversity differs for in utero and intrapartum transmission, and pointing the way to different strategies for interrupting viral transmission. They found that women transmitting HIV to their infants had significantly lower HIV strain diversity at delivery than nontransmitting women.

\section{Social/behavioral and public health sciences}

A growing diversity of subpopulations has been affected by $\mathrm{HIV}$, as shown by the increasing proportion of AIDS cases in California among African Americans, Latinos, women, and persons who inject drugs. Developing strategies for changing highly personal and often stigmatized behaviors across a growing number of subgroups pose a challenge for public health researchers. Participants in the annual meeting were given a number of insights into the nature of this challenge.

1. Can HIV risk be modified in socioeconomically disadvantaged populations?

Sharing of drugs and injection equipment is an important survival tactic among homeless IDUs observed in a participant-observation field study in San Francisco. IDUs who share drugs and injection equipment can expect to receive assistance in the future from those they assist, leading them to share resources with other injectors to protect against possible future isolation when they are confronted with an emergency shortfall of heroin. As a result, generosity and social group solidarity lead directly to health risk. The implications of such behaviors from a public health point of view are dire, although such acts are entirely rational to the IDUs in averting heroin withdrawal specifically and further impoverishment in general.

Low-income, disenfranchised African American men who have sex with men in the Tenderloin neighborhood 

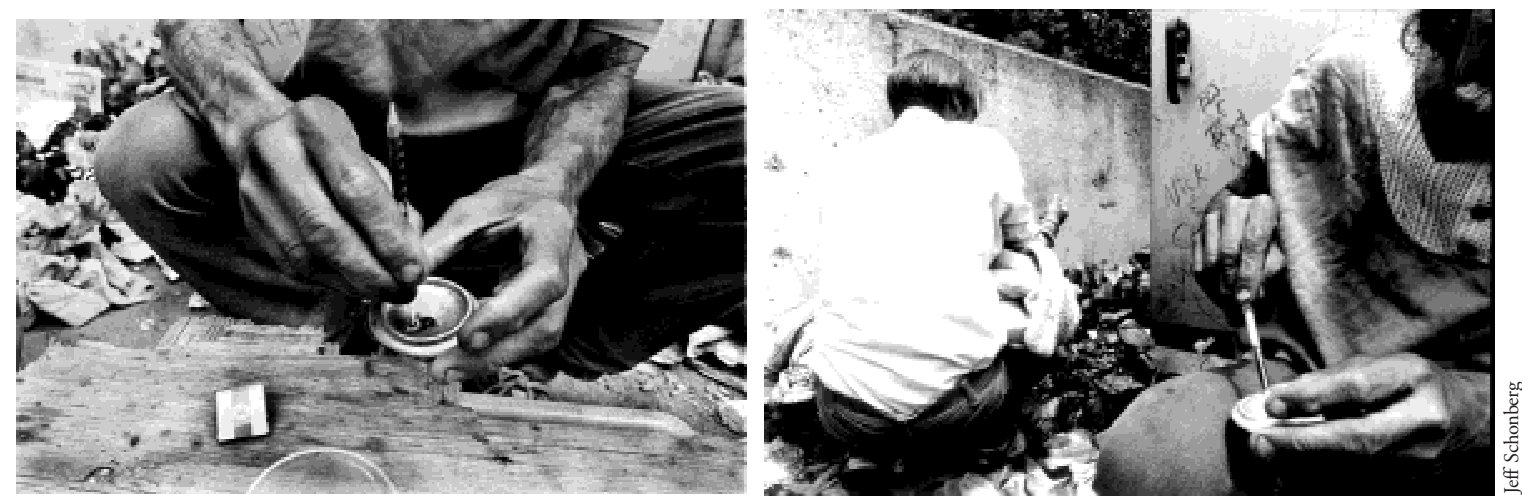

Sharing drugs and injection equipment is socially important to homeless drug misusers, despite its high risk. (Courtesy of Philippe Bourgois)

of San Francisco are engaging in high rates of unprotected anal intercourse, often without knowledge of their partner's serostatus. A convenience sample of these men $(\mathrm{n}=246)$ was recruited through flyers. At least a third of the men reported recent unprotected sex, and half reported some significant deficiency in knowledge of how HIV is sexually transmitted.

Social disadvantage is associated with STDs and HIV infection. In another low-income neighborhood in San Francisco, African American youth have disproportionately high rates of STDs. A community-based peer-led prevention program has just begun enrollment with the goal of increasing screening and treatment among this high-risk population.

Street youth are at high risk for hepatitis B and hepatitis C, as well as HIV. A San Francisco project used street-based sampling to recruit 310 IDUs younger than 30 years. Data were collected through interviews and serologic assays. Young female IDUs, despite shorter injection histories than men, were just as likely to be infected with HIV and viral hepatitis as male IDUs, suggesting that they are a group at particularly high risk of viral infection.

Transgendered male-to-female sex workers are a population at very high risk for HIV infection. Of 244 male-to-female transgendered people referred for study

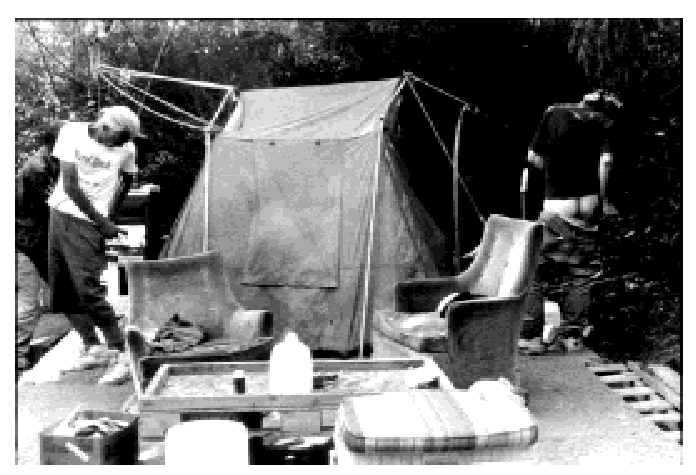

HIV risk remains high in economically disadvantaged groups. (Courtesy of Philippe Bourgois) by service agencies in Los Angeles, 58\% were sex workers. The workers were much more likely to engage in insertive and receptive anal sex and to inject nonhormonal drugs. But in a multivariate analysis, Latino ethnicity, less than 12 years of education, and younger than 30 years were more predictive of HIV seropositivity than risk behaviors.

2. Which interventions engage HIV-affected communities?

Several investigators reported promising findings related to HIV prevention among socially disadvantaged populations, documenting important developments in risk reduction initiated by at-risk groups. A study of white and Latino gay male youth found that the prevalence of unprotected sex with partners not identified as the respondent' boyfriend was relatively low. In an analysis of recent sexual activity, less than $10 \%$ of a sample of 40 gay youth reported unsafe sex with a person not identified as their boyfriend. Rates of unprotected sex with boyfriends, however, continued to be high. A survey of 549 men who have sex with men recruited in parks, dance clubs, and bars found that although alcohol and drug use were widespread in the young men, substance use was not associated with HIV risk behaviors.

Three studies focusing on IDUs have shown that many of those, referred to as "secondary exchangers," who attend syringe exchange programs exchange needles for other IDUs who do not use such programs. This practice emerges naturally out of existing relationships and is typically done out of friendship and altruism. A pilot study of 369 IDUs in a community-based sample found that secondary exchangers are willing to and are effective at disseminating HIV prevention messages to their social contacts.

3. What are the challenges to developing effective interventions?

The RESPECT-2 study is a randomized controlled trial taking place in 3 urban publicly funded STD clinics among patients seeking STD examinations. It will test the efficacy of rapid HIV testing and counseling in preventing 
the reoccurrence of STDs among clients of urban public health clinics. Although no outcome data are available to report, follow-up rates for 3-and 6-month interviews have been good: $87 \%$ and $79 \%$, respectively. Determining the efficacy of innovative HIV testing and counseling procedures in public health clinics is of growing importance. Yet, replicating a proven community-level prevention intervention outside of a research environment poses a number of problems. A case study of barriers to implementation of an HIV prevention program for young gay men in a community setting showed problems such as developing user-friendly technical assistance, a high staff turnover in the community-based agency charged with implementing the intervention, and the need for ongoing intensive training of front-line staff.

One of the most successful interventions presented at the meeting was one initiated by the Forensic AIDS Project team. The team provides care to HIV-positive inmates in the San Francisco County Jail system. A convenience sample of 53 HIV-positive inmates was recruited during a 6-month period in 1999. Participants were primarily men, 59\% were African American, and 30\% were white. The study found that a higher percentage of HIV-positive inmates are receiving antiretroviral therapy in jail than similar populations receiving care in community clinics. The investigators also found that antiviral resistance is low in this population. These findings underscore the desire among disadvantaged groups to receive care. Ironically, as the authors point out, the only place where socially dis- advantaged groups are guaranteed HIV treatment constitutionally is in jail.

\section{CONCLUSION}

An ongoing challenge for social and behavioral scientists and for society in general will be to develop strategies that reach the diverse populations at risk for HIV. The researchers at the 3rd Annual Conference on AIDS Research in California are aware of this challenge and are attempting to meet it in diverse and innovative ways.

\author{
Nancy E Moss \\ George Lemp \\ University of California, Universitywide AIDS Research Program
}

Ricky Bluthenthal

Center for HIV Identification, Prevention and Treatment Services, RAND Center on AIDS Research, Education and Services, Charles R Drew University

Mark A Goldsmith

Department of Medicine and Gladstone Institute, University of California, San Francisco

Richard Haubrich

Department of Infectious Diseases Treatment Center, University of California, San Diego

James 0 Kahn

Department of Medicine, University of California, San Francisco and San Francisco General Hospital.

\section{Babies and cream cheese}

While on call one night, I was paged by a nurse about a neonate. The nurse asked if I could come and examine the baby, since the father was extremely worried. The reason? He had dropped cream cheese on the baby's head. Naturally, my first clinical question over the phone was, "Regular, soft, or whipped?" I made my way to the scene, where the anxious parent filled in the details. "I was trying to spread the cream cheese on a bagel," he said, "but it slipped from the knife." At this point, I had to use every ounce of energy to prevent from breaking out into uncontrollable laughter. I held myself back, however, and proceeded to assess the mechanism of injury. Fortunately, it was only soft cream cheese, and the baby survived the ordeal unscathed. I assured the father that the baby was fine, and that compared to the trauma of a normal spontaneous vaginal delivery, the baby's encounter with the fermented milk would not be a problem. I also told him that for this exact reason, as part of our normal anticipatory guidance to parents of newborns, we only recommend having whipped cream cheese in the house in order to prevent any serious injury to newborns. 